\title{
Male Rat's Liver Weight Molding Based on Grey Theory and RBF Neural Network
}

\author{
Jianyang $\operatorname{Lin}^{1 *}$, Jinjie $\mathrm{Xu}^{1}$ and Zhien $\mathrm{Sun}^{2}$ \\ 1. Department of Pharmacy, the First Hospital of China Medical University, Shenyang City, China \\ 2. School of Automobile and Transportation, Shenyang Ligong University, Shenyang City, China \\ *Corresponding author
}

\begin{abstract}
In order to research the relationship between male rat's liver weight and their body weight, Grey theory method and Radial Basis Function neural network method were used to model the liver related data in male rats. First of all, the sample standard deviations of the liver weight mean value were obtained by using Grey theory method. What's more, validated liver weight data in each group was in accord with normal distribution and calculated the upper and lower limits of liver weight distribution. The RBF neural network method was applied to fit the upper and lower limits of liver weight distribution and mean values in software R2011b MATLAB environment. Final fitting result which has practical value can be used to verify actual liver weight show relationship between liver weight and body weight, and then it can cut down test cost and cycle.

Keyword-liver weight of male rat; Grey theory; RBF neural network; MATLAB
\end{abstract}

\section{INTRODUCTION}

The organ weight data of experimental animals is an important basis index for biomedical research. It not only has a guiding significance to the normal physiological state of animals, but also an important reference index in the non clinical study of drugs[1]. Therefore, it is necessary to establish the mathematical model of male rats' liver weight.

There are 30 groups of male rats in this experiment; each group contains 17 male rats. We anatomized and measured liver weight after the body weight of rats measured. In total, 510 groups' discrete data of liver weight and body weight were obtained. According to the actual measurement results predicted, we find there is certain relationship between liver weight and body weight in rats. We use some methods to find out the relationship between liver weight and body weight from experimental data, and then establish the mathematical model of male rats' liver weight.

Using MATLAB software to process experimental data can greatly reduce the error caused by the calculation and plotting. It can be selected desired function according to the specific circumstances when fit curve by use of MATLAB curve fitting toolbox, so as to obtain relatively accurate results[2]. Grey theory and RBF neural network theory were mainly applied when processed liver weight data in MATLAB R2011b. Related content are as follows.

\section{A. Related Content about Grey Theory}

Accumulation generation operation is the most important data processing method in grey relational analysis. Through accumulation of generating operations, the arbitrary non negative sequence and swing sequence can be converted into incremental sequence[3].

Suppose there is a set of measured data sequences:

$\mathrm{X}=(\mathrm{x}(\mathrm{k}) \mid \mathrm{k}=1,2 \cdots, \mathrm{n})$. The data sequence $\mathrm{X}$ is converted to $\mathrm{X}^{0}$ after arranged from small to large. We get

$$
\begin{gathered}
X^{0}=\left(x^{(0)}(k) \mid k=1,2, \cdots, n\right)= \\
\left(x^{(0)}(1), x^{(0)}(2), \cdots, x^{(0)}(n)\right.
\end{gathered}
$$

Where, $x^{0}(k) \leq x^{(0)}(k+1), k=1,2, \cdots, n-1$.

The new $X^{(1)}$ is result of that $X^{0}$ through one time Accumulated Generation Operation (1-AGO), that is

$$
\begin{gathered}
X^{1}=\left(x^{(1)}(k) \mid k=1,2, \cdots, n\right)= \\
\left(x^{(1)}(1), x^{(1)}(2), \cdots, x^{(1)}(n)\right)=\left(x^{(0)}(1), x^{(0)}(1)+\right. \\
\left.x^{(0)}(2), \cdots, x^{(0)}(1)+x^{(0)}(2)+\cdots+x^{(0)}(n)\right)
\end{gathered}
$$

The irregular original data are showing growth regularity after cumulative generation. Original measurement sequence and accumulated measuring sequence are all linear because of there is no measurement error in ideal measurement process. The $\Delta(\mathrm{k})$ which is maximum vertical distance between measured value and ideal value can be used to describe dispersion of data.

We define:

$$
\begin{aligned}
\Delta(\mathrm{k}) & =\frac{\mathrm{x}^{(1)}(\mathrm{n})}{\mathrm{n}} \mathrm{k}-\mathrm{x}^{(1)}(\mathrm{k}) \\
\Delta_{\max } & =\max (\Delta(1), \Delta(2), \cdots, \Delta(\mathrm{n}))
\end{aligned}
$$

The curved cumulative curve is becoming increasingly curved with greater standard deviation of measured data samples.

Define standard deviation:

$$
\mathrm{s}=\mathrm{c} \cdot \Delta_{\max } / \mathrm{n}
$$

Where, $\mathrm{c}$ is grey constant coefficient; $\mathrm{n}$ is the number of samples for measurement data. Often take $c=2.50$ to calculate the standard deviation[4]. 


\section{B. Neural Network Theory}

Artificial neural network (ANN) is a kind of mathematical model which simulated biological neural network for information processing. It also can be called neural network for short[5]. Its essence is a network which is interconnected mathematical nodes or neurons. It can simulate complex functional relationships, especially suitable for problems involving multiple parameters and nonlinear interpolation[6].

RBF neural network is a kind of three layers forward network which similar to Multilayer forward network. First layer is input layer which is composed of the signal source node. Second layer is the hidden layer, and the nodes number in hidden layer is up to need of the problem description. The transform function of the neuron in the hidden layer (radial basis function) is nonlinear function which radial symmetry and attenuation of the center point. It is local response function. Third layer is the output layer which responds to the input mode.

The RBF neural network has been successfully applied in many fields because of its superior capability about function approximation and simple construct. The representative applications include system identification, face recognition, prediction and antenna design etc. Typical structure of RBF neural network is shown in Figure I.

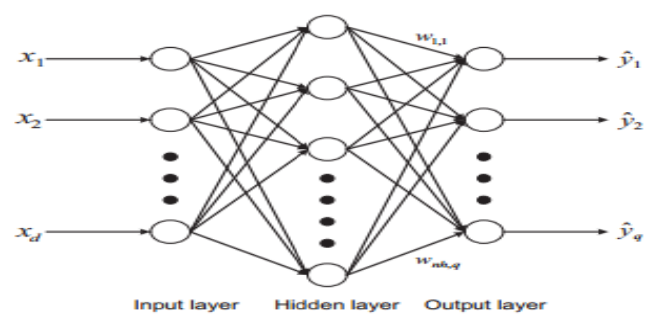

FIGURE I. TYPICAL STRUCTURE OF RBF

Radial basis function which usually defined as the monotonic function of Euclidean distance between any points in space to a center is used in node activation function of Radial Basis Function neural network. Frequently used radial basis function is Gaussian Function. Activation function of radial basis function neural network can be expressed as follow.

$$
R\left(x_{p}-c_{i}\right)=\exp \left(-\frac{1}{2 \sigma^{2}}\left\|x_{p}-c_{i}\right\|^{2}\right)
$$

Where, $\left\|x_{p}-c_{i}\right\|$ is Euclidean norm; $c_{i}$ is center of the Gaussian function; $\sigma$ is Variance of Gaussian function.

According to the different methods of radial basis function center selection, RBF neural network has a variety of learning methods. For instance, random select central method, selforganizing selection method, supervision of select central method and the orthogonal least square method etc. Self organizing selection center method consists of two phases which introduced as follows: one of them is self organizing learning phase which Figure out center and variance of hidden layer basis function, the other is supervised learning phase and it is to solve weights from hidden layer to output layer.
Detailed steps of this learning algorithm are as follows.

First step: to solve basis function center c based on $\mathrm{K}-$ mean clustering method.

1. Network initialization: random select $\mathrm{h}$ training samples as cluster center $c_{i}(i=1,2, \cdots, h)$.

2. Input training samples are grouped according to the nearest neighbor rule: assign $\mathrm{x}_{\mathrm{p}}$ to each cluster set of input samples $p(p=1,2, \cdots, P)$ according to Euclidean distance between $x_{p}$ and $c_{i}$.

3. Adjust cluster center again: calculate average value of training samples in every clustering ensemble $p$. That is new cluster center $c_{i}$. If there is no change in new cluster center, $c_{i}$ is basis function center of the RBF neural network finally. Otherwise, return to 2 and to solve next round of the center.

Second step: to solve variance $\sigma_{i}$.

Variance $\sigma_{i}$ can solve as follows if basis function of RBF neural network is Gaussian function.

$$
\sigma_{i}=\frac{c_{\max }}{\sqrt{2 h}} \quad i=1,2, \cdots, h
$$

Where, $c_{\max }$ is the maximum distance between selected center.

Third step: calculate weights between hidden layer and output layer.

Connection weights between the hidden layer and the output layer can be calculated by the least square method directly and calculating formula as follows:

$$
\mathrm{w}=\exp \left(\frac{\mathrm{h}}{\mathrm{c}_{\max }^{2}}\left\|\mathrm{x}_{\mathrm{p}}-\mathrm{c}_{\mathrm{i}}\right\|^{2}\right)
$$

Where, $\mathrm{i}=1,2, \cdots, \mathrm{h} ; \mathrm{p}=1,2, \cdots, \mathrm{P}$

The corresponding function of RBF neural network in software MATLAB as follows.

$$
[\text { net, } \mathrm{tr}]=\operatorname{newrb}(\mathrm{P}, \mathrm{T}, \mathrm{GOAL}, \mathrm{SPEAD}, \mathrm{MN}, \mathrm{DF})
$$

Where, $\mathrm{P}$ is $\mathrm{R}^{*} \mathrm{Q}$ dimensional matrix consists of $\mathrm{Q}$ group input vector; $\mathrm{T}$ is $\mathrm{S}^{*} \mathrm{Q}$ dimensional matrix consists of $\mathrm{Q}$ group target classification vector; GOAL is Mean Squared Error Goal and it defaults to 0.0; SPEAD is expansion velocity of radial basis function and it defaults to $1 ; \mathrm{MN}$ is Maximum number of neurons and it defaults to Q; DF is number of neurons added between the two display and it defaults to 25; net is return value, one RBF neural network; TR is return value, training record[7-9].

\section{DATA PROCESSING}

\section{A. Calculate Arithmetic Mean and Gray Standard Deviation}

Mean function in software MATLAB was used to calculate arithmetic mean value of body weight and liver weight in each group, and then using grey theory method to calculate standard deviation of liver weight. Calculation results are shown in 
Table I. Where, $\mu_{1}$ stands for body weight arithmetic average value of rats in each group. $\mu_{2}$ stands for

arithmetic average value of liver weight. $\sigma$ stands for gray standard deviation of liver weight mean value.

TABLE I. MEAN VALUE OF BODY WEIGHT AND LIVER WEIGHT, GRAY STANDARD DEVIATION OF LIVER WEIGHT

\begin{tabular}{|l|l|l|l|l|l|l|l|l|l|l|}
\hline NUMBEWR & $\mathbf{1}$ & $\mathbf{2}$ & $\mathbf{3}$ & $\mathbf{4}$ & $\mathbf{5}$ & $\mathbf{6}$ & $\mathbf{7}$ & $\mathbf{8}$ & $\mathbf{9}$ & $\mathbf{1 0}$ \\
\hline $\boldsymbol{\mu}_{\mathbf{1}} / \mathbf{g}$ & 62.92 & 101.44 & 151.29 & 203.10 & 241.34 & 271.49 & 296.46 & 321.64 & 334.64 & 347.29 \\
$\boldsymbol{\mu}_{2} / \mathbf{g}$ & 2.845 & 4.375 & 7.286 & 8.574 & 10.209 & 10.587 & 12.212 & 13.336 & 12.800 & 13.950 \\
$\boldsymbol{\sigma} / \mathbf{g}$ & 0.208 & 0.399 & 0.728 & 1.439 & 2.287 & 1.781 & 2.187 & 1.648 & 1.351 & 2.059 \\
\hline NUMBER & $\mathbf{1 1}$ & $\mathbf{1 2}$ & $\mathbf{1 3}$ & $\mathbf{1 4}$ & $\mathbf{1 5}$ & $\mathbf{1 6}$ & $\mathbf{1 7}$ & $\mathbf{1 8}$ & $\mathbf{1 9}$ & $\mathbf{2 0}$ \\
\hline $\boldsymbol{\mu}_{\mathbf{1}} / \mathbf{g}$ & 364.94 & 378.40 & 394.54 & 407.57 & 422.89 & 433.88 & 445.06 & 456.98 & 466.87 & 476.71 \\
$\boldsymbol{\mu}_{\mathbf{2}} / \mathbf{g}$ & 14.339 & 15.480 & 16.810 & 17.088 & 16.968 & 17.057 & 17.529 & 17.940 & 18.535 & 19.307 \\
$\boldsymbol{\sigma} / \mathbf{g}$ & 2.119 & 2.127 & 1.784 & 1.766 & 2.155 & 2.492 & 1.412 & 2.478 & 1.664 & 2.316 \\
\hline NUMBER & $\mathbf{2 1}$ & $\mathbf{2 2}$ & $\mathbf{2 3}$ & $\mathbf{2 4}$ & $\mathbf{2 5}$ & $\mathbf{2 6}$ & $\mathbf{2 7}$ & $\mathbf{2 8}$ & $\mathbf{2 9}$ & $\mathbf{3 0}$ \\
\hline $\boldsymbol{\mu}_{\mathbf{1}} / \mathbf{g}$ & 485.10 & 495.61 & 507.18 & 523.03 & 537.32 & 549.15 & 565.34 & 584.76 & 601.73 & 635.67 \\
$\boldsymbol{\mu}_{2} / \mathbf{g}$ & 19.133 & 19.085 & 18.978 & 19.398 & 19.890 & 20.092 & 20.441 & 20.934 & 20.526 & 22.255 \\
$\boldsymbol{\sigma} / \mathbf{g}$ & 2.796 & 2.497 & 1.676 & 2.488 & 2.541 & 1.748 & 1.565 & 2.265 & 2.362 & 1.811 \\
\hline
\end{tabular}

TABLE II. $\quad$ DISTRIBUTION INTERVAL OF LIVER WEIGHT

\begin{tabular}{|l|l|l|l|l|l|l|l|l|l|l|}
\hline NUMBER & $\mathbf{1}$ & $\mathbf{2}$ & $\mathbf{3}$ & $\mathbf{4}$ & $\mathbf{5}$ & $\mathbf{6}$ & $\mathbf{7}$ & $\mathbf{8}$ & $\mathbf{9}$ & $\mathbf{1 0}$ \\
\hline$\left(\boldsymbol{\mu}_{\mathbf{2}}+\mathbf{3} \boldsymbol{\sigma}\right) / \mathbf{g}$ & 3.469 & 5.572 & 9.470 & 12.891 & 17.070 & 15.930 & 18.773 & 18.280 & 16.853 & 20.127 \\
$\left(\boldsymbol{\mu}_{\mathbf{2}}+\mathbf{3} \boldsymbol{\sigma}\right) / \mathbf{g}$ & 2.221 & 3.178 & 5.102 & 4.257 & 3.348 & 5.244 & 5.561 & 8.392 & 8.747 & 7.773 \\
\hline NUMBER & $\mathbf{1 1}$ & $\mathbf{1 2}$ & $\mathbf{1 3}$ & $\mathbf{1 4}$ & $\mathbf{1 5}$ & $\mathbf{1 6}$ & $\mathbf{1 7}$ & $\mathbf{1 8}$ & $\mathbf{1 9}$ & $\mathbf{2 0}$ \\
\hline$\left(\boldsymbol{\mu}_{2}+\mathbf{3} \boldsymbol{\sigma}\right) / \mathbf{g}$ & 20.696 & 21.861 & 22.162 & 22.386 & 23.433 & 24.533 & 21.765 & 25.374 & 23.527 & 26.255 \\
$\left(\boldsymbol{\mu}_{\mathbf{2}}+\mathbf{3} \boldsymbol{\sigma}\right) / \mathbf{g}$ & 7.982 & 9.099 & 11.458 & 11.790 & 10.503 & 9.581 & 13.293 & 10.506 & 13.543 & 12.359 \\
\hline $\mathbf{N U M B E R}$ & $\mathbf{2 1}$ & $\mathbf{2 2}$ & $\mathbf{2 3}$ & $\mathbf{2 4}$ & $\mathbf{2 5}$ & $\mathbf{2 6}$ & $\mathbf{2 7}$ & $\mathbf{2 8}$ & $\mathbf{2 9}$ & $\mathbf{3 0}$ \\
\hline$\left(\boldsymbol{\mu}_{\mathbf{2}}+\mathbf{3} \boldsymbol{\sigma}\right) / \mathbf{g}$ & 27.521 & 26.576 & 24.006 & 26.862 & 27.513 & 25.336 & 25.136 & 27.729 & 27.612 & 27.688 \\
$\left(\boldsymbol{\mu}_{2}+\mathbf{3} \boldsymbol{\sigma}\right) / \mathbf{g}$ & 10.745 & 11.594 & 13.950 & 11.934 & 12.267 & 14.848 & 15.746 & 14.139 & 13.440 & 16.822 \\
\hline
\end{tabular}

\section{B. Normality Test of Data}

Data normality test is an important part in course of data processing. Normal probability paper test was used to test whether liver weight in each group was consistent with the normal distribution. The normal distribution probability map about the 17 data in each group was drawn by the normplot function in MATLAB function library. Selected 10th group randomly to test and the test results are shown in Figure. II. Normality test of other groups are similar to them and don't list one by one because of limited space in this paper.

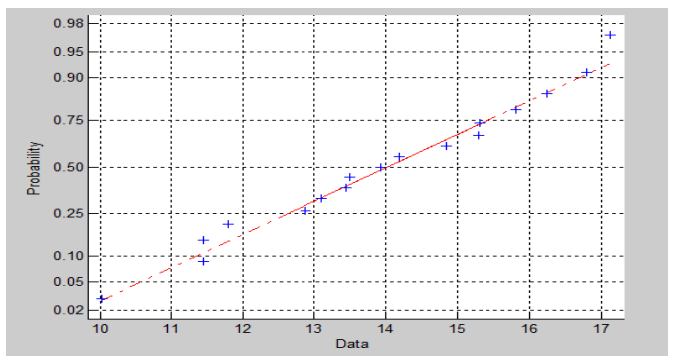

FIGURE II. NORMAL TEST RESULTS OF THE 10TH GROUP

Each set of data distributed in a straight line basically can be seen from the test chart. The results are in accord with the normal distribution, and then next data analysis can be carried out.

\section{Liver Weight Distribution Interval and Fitting Them}

Under normal distribution condition, probability of random variable $\mathrm{x}$ falls in interval $\sigma$ which is result of $\mu$ added and subtracted different multiples of $\sigma$. For instance,

$$
\begin{aligned}
& \mathrm{P}(\mu-1.96 \sigma \leq \mathrm{x}<\mu+1.96 \sigma)=0.9 \\
& \mathrm{P}(\mu-2.58 \sigma \leq \mathrm{x}<\mu+2.58 \sigma)=0.99 \\
& \mathrm{P}(\mu-3 \sigma \leq \mathrm{x}<\mu+3 \sigma)=0.9973
\end{aligned}
$$

Probability of random variable $x$ falls between $\mu \pm 3 \sigma$ is 0.9973 by above probability formula[10]. Therefore, using formula $\mu_{2} \pm 3 \sigma$ to calculate distribution of liver weight range can cover most of the data and results are more accurately, as shown in Table II. It can be seen from Table II that rat's liver weight distribution showed a gradual upward trend.

It needs to be regarded as a whole discrete observation data and turned into continuous function, in order to analyze these data dynamically, digging out more useful information from data, showing the deep regularity. Data fitting is the main method of data function. The approximate function obtained by fitting data which can better reflect observation data does not require that approximate function must be the same as the observation data at each observation point, but the overall error is smaller[11].

What follows is using RBF neural network method to fit the upper and lower limits of liver weight distribution and mean value of liver weight. Curve of error performance about liver weight mean value is shown in Figure. III. Curve of error performance about liver weight distribution interval are also ideal and don't list one by one because of limited space in this paper. It can get better fitting effect when the value of SPREAD is 20 after a series of analysis and comparison. Final fitting result is shown in Figure. IV. 


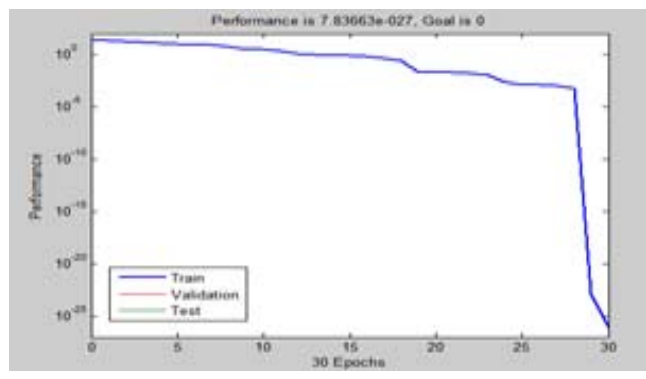

FIGURE III. ERROR PERFORMANCE ABOUT LIVER WEIGHT MEAN VALUE

The relationship between male rats liver weight and body weight can be seen directly from Figure. IV.

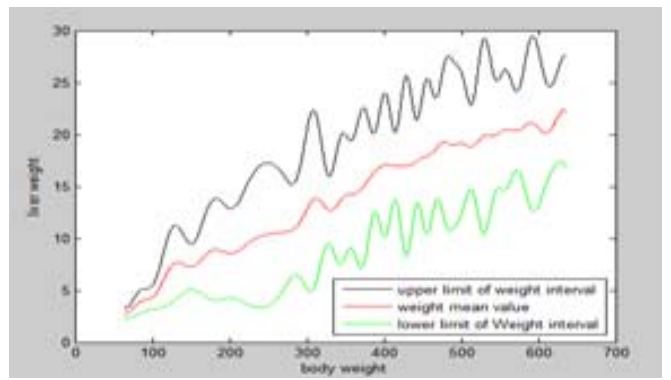

FIGURE IV. FITING RESULTS OF RBF NEURAL NETWORK

\section{CONCLUSION}

In this paper, used grey theory method to process the experimental male rats' data of liver weight data, and then established mathematical model of male rats' liver weight and body weight by using RBF neural network method to fit data in software R2011b MATLAB environment. Following conclusions can be obtained after the above analysis.

(1) RBF neural network method is suitable for male rats' liver weight data modeling. The obtained mathematical model is very accurate which have high degree of agreement with the original measurement data and it can be adjusted according to the actual needs.

(2) A series of male rats' liver weight interval distribution were completed and the results can be used to verify whether liver weight repeatability test data are accurate. It can provide reference for future research. In addition, it can cut down test expenses or cycle and decrease cost by reducing consumption of rats for measuring actual liver weight.

(3) The fitting result curve can be used as a standard to verify whether the male rat's liver is abnormal. Liver weight measured in actual medical experiment does not accord with this fitting result, that is, actual liver weight data are located outside the upper and lower bounds of the distribution interval. Then the liver weight may be abnormal and provide a theoretical basis for further disease detection.

\section{ACKNOWLEDGMENT}

This work was supported by National Natural Science Foundation of China under Grant 81302841 and University Outstanding Talent Support Plan Foundation of Liaoning Province under Grant LJQ2014086.

\section{REFERENCES}

[1] DONG Yan-sheng, YIN Ji-ye, CHEN Zhang. Establishment and application of the normal reference values of organ/body coefficient in SD rats[J]. MILITARY MEDICAL SCIENCES, 2012, 36(5) 351-353

[2] ZHAO Feng, Development of Experiment Data Processing Based on Matlab[J].Development \& Innovation of Machinery \& Electrical Products, 2012, 25(2):73

[3] Jianyang Lin, Hui Zhou, Pinellia Quality Assessment based on Grey Uncertainty and Rayleigh Distribution Similarity $[\mathrm{J}]$. International Journal of Advancements in Computing Technology, 2012, 4(13):115116

[4] Wang Zhong-yu, Xia Xin-tao, Zhu Jian-min. Non-statistics theory and engineering apply[M]. Beijing: Science Press, 2005: 22-27

[5] WANG Shuti, BA Yin-liang, GUO Zeng-bo. Electronic controlled engine fault diagnosis based on BP neural network [J]. Modern Electronics Technique, 2015, 38(9):128

[6] W. Sha, K.L. Edwards. The use of artificial neural networks in materials science based research [J]. Materials and Design, 2007(28):1747-1752

[7] Michael M. Li, Brijesh Verma, Nonlinear curve fitting to stopping power data using RBF neural networks [J]. Expert Systems With Applications. 2016(45):161-171

[8] WANG Xiao-chuan, SHI Feng, YU Lei. 43 cases analysis of MATLAB neural network [M]. Beijing: Beihang University Press. 2013:59-62

[9] Yiran Shi , Ding-Li Yu, Yantao Tian,etc. Air-fuel ratio prediction and NMPC for SI engines with modified Volterra model and RBF network[J] Engineering Applications of Artificial Intelligence 2015, 45:313-324

[10] LI Zhi-xi, DU Shuang-kui. Experimental optimization design and statistical analysis [M].Beijing: Science Press, 2010:17-18

[11] WANG Li, WANG Wen-jian, JIANG Gao-xia. Optimization for Smoothing Parameter in Process of Data Fitting [J]. Computer Science, 2015, 42(9): 226-229 